\title{
Experimental studies for the additive manufacturing of continuous fiber reinforced composites using UV-curing thermosets
}

\author{
Eckart Kunze, Michael Müller-Pabel, Oliver Weißenborn, Ron Luft, Johann Faust, Sirko Geller and Maik Gude
}

Eckart Kunze. Technische Universität Dresden - Institute of Lightweight Engineering and Polymer Technology, Holbeinstr. 3, D-01307 Dresden, Germany. Corresponding author: Kunze, Eckart. E-mail address: Eckart.kunze@tu-dresden.de

Michael Müller-Pabel. Technische Universität Dresden - Institute of Lightweight Engineering and Polymer Technology,

Holbeinstr. 3, D-01307 Dresden, Germany

Oliver Weißenborn. Technische Universität Dresden - Institute of Lightweight Engineering and Polymer Technology, Holbeinstr.

3, D-01307 Dresden, Germany

Ron Luft. Technische Universität Dresden - Institute of Lightweight Engineering and Polymer Technology, Holbeinstr. 3, D-01307

Dresden, Germany

Johann Faust. Technische Universität Dresden - Institute of Lightweight Engineering and Polymer Technology, Holbeinstr. 3,

D-01307 Dresden, Germany

Sirko Geller. Technische Universität Dresden - Institute of Lightweight Engineering and Polymer Technology, Holbeinstr. 3,

D-01307 Dresden, Germany

Maik Gude. Technische Universität Dresden - Institute of Lightweight Engineering and Polymer Technology, Holbeinstr. 3, D-01307

Dresden, Germany

\begin{abstract}
The economical production of lightweight structures with tailor-made properties and load-adapted geometry is limited using conventional technologies. Additive manufacturing processes offer a high potential to meet these requirements, where the established solutions are based primarily on thermoplastics matrix systems. From a process-technological point of view, thermoplastics enable simplified processing, but only a limited range of applications for high-performance components. These limitations are due to their comparatively low heat resistance, low melting temperatures and limited adhesion to embedded reinforcing fibers. In contrast, thermosets show high potential for realization of high-performance lightweight structures with adaptable properties. The present work employs a UV-curing thermoset resin for the impregnation of a continuous filament strand for 3D printing. The main challenge is to reconcile the crosslinking reaction of the thermoset and the process velocity during impregnation and cure. The liquid polymer must provide low initial viscosity to impregnate the filaments and a sufficiently high cure rate and dimensional stability after discharge from the print head to ensure sufficient bonding strength to the substrate. To demonstrate feasibility, a prototypic print head with UV-LED activation was designed and implemented. With a robot-guided printing platform, the 3D-deposition of continuous fiber-reinforcements without additional supporting structures can be realized. To derive initial process parameters, reaction and thermos-mechanical properties are determined by rheometer measurements. Impregnation and cure behavior of the glass fiber reinforced resin is investigated. The presented results provide a reliable process window and a straightforward process monitoring method for further enhancement of the conceived 3D printing process.
\end{abstract}

Keywords. Additive Manufacturing, UV-Curing, Thermosets, Continuous Fiber Reinforcement

\section{Introduction}

Sustainable and efficient use of existing resources and the increasing demand for high-quality customizable products represent major future challenges for manufacturing industry. In this context, additive manufacturing with fiberreinforced polymers (FRP) offers both, individualized lightweight structures with tailor-made properties and loadadapted geometry and economical production with no or little waste [1]. Most of the currently available 3D printing technologies with polymeric materials rely on thermoplastic matrix materials [2-4]. From a process-technological 
Experimental studies for the additive manufacturing of continuous fiber reinforced comp...

point of view, thermoplastics enable simplified processing due to their hot-melt capability and a range of readily available pre-impregnated continuous fibers [5,6]. Also in-nozzle impregnation was reported [7].

In order to enable flexible realization of lightweight structures with high thermo-mechanical properties, the use of thermoset matrix materials is a promising approach for additive manufacturing. Thermosets provide high glass transition temperatures, excellent adhesion properties and low viscosity prior to cure. Additionally, adaptable properties can be achieved by varying reaction components and cure conditions. Conventional processing of thermoset matrix materials suffers from long curing times ranging from some minutes to hours, which is unsuitable for 3D printing. To circumvent this problem, radiation curable resins can be used instead of thermally activated formulations. Especially ultraviolet (UV) light activated resins provide fast curing without requiring additional heat input.

State of the art 3D printing technologies using UV curable resins are stereolithography (SLA) and digital light processing (DLP). With both processes, 3D-structures are built up in layers within a vat of resin. However, this approach does not allow the integration of continuous fiber reinforcement. Some robot-based applications are being developed that combine UV curable resins and continuous fibers for 3D printing [8,9]. A process similar to a thermoplastic 3Dprinting process is known from [10], which is based on a desktop-like 3D-printer that is used to coextrude a thermoset embedded fiber strand within a thermoplastic matrix.

The scope of this research is to present a methodical approach for a 3D printing process that combines a UV curing resin and continuous fiber reinforcement. For this purpose, the cure behavior of a UV activated epoxy resin is analyzed in order to define a process window for impregnation, curing and placement. A highly integrative print head is designed that allows fast production of FRP layers with flexible fiber orientation. Furthermore, a manufacturing cell is conceived and implemented.

\section{Materials and Methods}

\subsection{Materials}

For this study, a photo-curable resin system supplied by Sartomer Arkema Group was chosen. The base component S105 is a cycloaliphatic epoxy resin providing low initial viscosity. UviCure S130 is used as reactive diluent and cure accelerator. Speedcure 976 serves as cationic photoinitiator with a light absorption maximum between a wavelength of 279 to $291 \mathrm{~nm}$. All three components are mixed to a ratio of 100:10:3 pbw. Due to the cationic reaction mechanism, the activated resin is expected to show dark-cure and thermal post-cure behavior. To initiate photo-curing, a battery powered UV light source Convoy S2 was used providing an emission maximum at a wavelength $365 \mathrm{~nm}$ and a radiant flux of approximately $1100 \mathrm{~mW}$ at a forward current of $\mathrm{IF}_{\mathrm{F}}=700 \mathrm{~mA}$. It contains a NCSU276AT LED from Nichia and an AMC 7135 LED driver unit. A glass fiber roving EC 900-350 from P-D Glasseiden GmbH was selected as fiber material. It has a linear density of 900 tex and approx. 1.5k filaments with a diameter of $17 \mu \mathrm{m}$.

\subsection{Methods}

A MCR502 rheometer device (Anton Paar $\mathrm{GmbH}$ ) was used for characterization of both neat resin and fiber reinforced material. According to the specific cure-state and sample geometries, different setups and fixtures were utilized, see Fig. 1.

The plate-plate mode was used in combination with the Convoy S2 light source and a transparent bottom plate in order to analyze initial viscosity and photo-cure behavior at isothermal temperatures from room temperature to $100{ }^{\circ} \mathrm{C}$. An oscillating shear deformation with an amplitude of $0.5 \%$ and a frequency of $5 \mathrm{~Hz}$ was applied. The dynamic modulus was continuously evaluated throughout the liquid-solid-transition associated with gelation. The plate diameter was 15 
$\mathrm{mm}$ and the gap was set to $0.2 \mathrm{~mm}$. A solid rectangular fixture (SRF) was employed for dynamic-mechanical analysis (DMA) of a neat resin sample that was pre-photo- cured in an open silicone mold providing a nearly rectangular cross-section of $4 \mathrm{~mm} \times 1 \mathrm{~mm}$ and a specimen length of $40 \mathrm{~mm}$. The shear deformation amplitude was set to $0.01 \%$ and the frequency to $1 \mathrm{~Hz}$. A heating rate of $4 \mathrm{~K} / \mathrm{min}$ was applied from room temperature to $175^{\circ} \mathrm{C}$. In order to gain information on possible post-cure effects, the same specimen was analyzed in a second measurement. Finally, a solid circular fixture (SCF) with a diameter of $1 \mathrm{~mm}$ and a gap distance between 10 and $15 \mathrm{~mm}$ was used to perform DMA measurements on impregnated fiber reinforced composite specimens originating from the developed printing process. Again, pre-cured and post-cured stage were compared by performing two consecutive measurements.
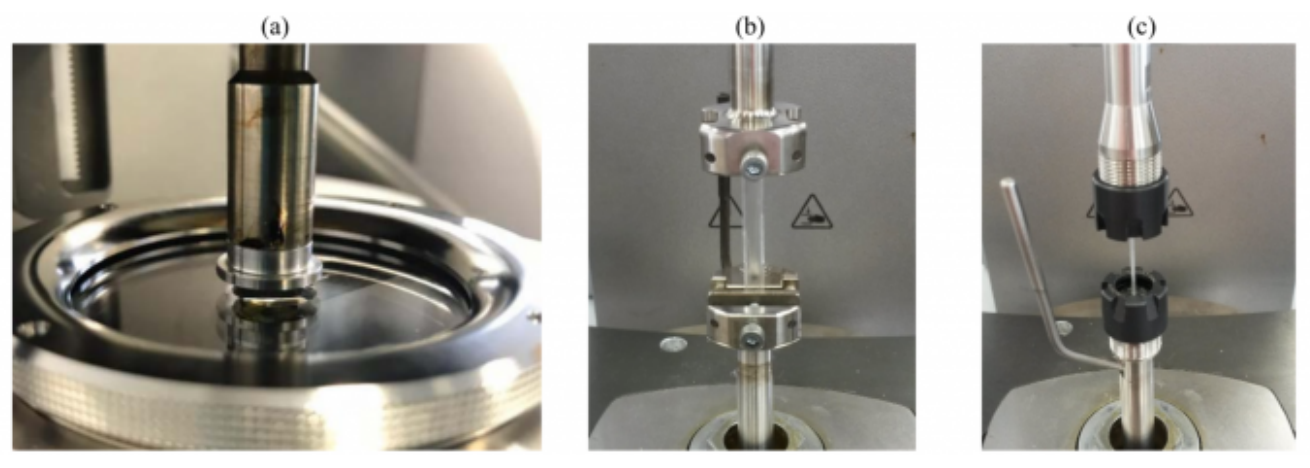

Fig. 1. Test setups for rheological and DMA experiments: (a) UV-rheology with uncured resin, (b) SRF with neat resin and (c) SCF with composite.

During the printing process, a VarioCam HD thermal imaging camera from Jenoptik was used to monitor the temperature profile of the impregnated fiber strand before, during and after exposure to the UV light source. The measurements were carried out at a test stand setup (see section 2.3 and Fig. 2 a). The camera was mounted on a tripod with a viewing direction perpendicular to the roving. A sampling rate of $20 \mathrm{~Hz}$ was used. Thermal profiles are analyzed along several vertical lines across the roving width. The measured temperatures were averaged to prevent temperature discontinuities.

\subsection{Functional principle of the printing head}

A test stand was designed to qualify the developed printing process apart from an industrial robot. The test stand comprises the actual print head and a temporary unit for pulling and winding up the roving. The print head consists of the commercially available Viscotec DuoP 4/4 metering machine, the self-developed impregnation tool, the LED light source, the printing nozzle and a carrier plate to connect all components. The metering machine is capable of mixing two components and of precise dosing of volume flow rates down to $0.002 \mathrm{ml} / \mathrm{s}$. For this study, its two cartridges are filled with the premixed epoxy resin, while the metering machine is used for precise metering only.

The developed steel impregnation tool consists of a resin and a fiber inlet, and an outlet for the impregnated roving. The metering machine is connected with a static mixer to the resin inlet. The roving is pulled from a reel above the tool into the fiber inlet, which consists of a ceramic nozzle to reduce friction and prevent fiber damage. For the same reason, all external yarn guides are made of ceramic material. Within the tool, the resin is led to an enlarged chamber to impregnate the roving. The fiber outlet diameter is $1 \mathrm{~mm}$. The impregnated roving passes a circular light source and exits the print head through an interchangeable printing nozzle. For the experiments, the external light source Convoy S2 was applied and the impregnated roving was exposed after the ceramic nozzle. To simulate the print process with a stationary print head, a rotating mandrel with a diameter of $125 \mathrm{~mm}$ replaces the moving print bed. The tube's 
Experimental studies for the additive manufacturing of continuous fiber reinforced comp...

rotational speed can be matched to the volume flow rate of the metering machine.

To investigate the influence of different process parameters on impregnation and curing (see Table 1), thermographic images and samples for DMA measurement were taken from the location marked in (Fig. 2b). The parameter 'UV light intensity' was adjusted by varying the distance of the light source to the roving. Printing velocity was adjusted by varying the rotational speed of the mandrel.

\begin{tabular}{lll}
\hline Test set up & $\begin{array}{l}\text { Distance of light source } \\
\text { to roving }[\mathrm{mm}]\end{array}$ & $\begin{array}{l}\text { Printing velocity }[\mathrm{mm} / \mathrm{s}] / \\
\text { Resin flow rate }[\mathrm{ml} / \mathrm{s}]\end{array}$ \\
\hline Variant 1 (Fig. 2) & 7.5 & $4.6 / 0.002$ \\
\hline & 15 & $11.5 / 0.005$ \\
\hline & 30 & $18.5 / 0.008$ \\
\hline
\end{tabular}

Table 1. Levels for variation of the different test parameters.

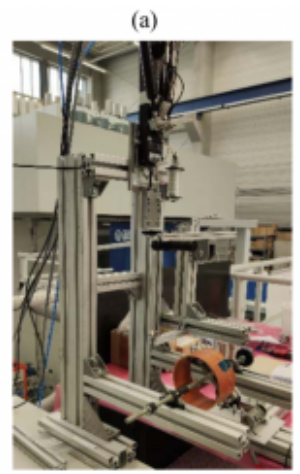

(b)

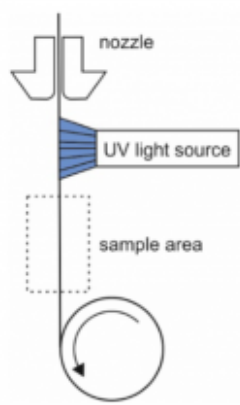

(c)

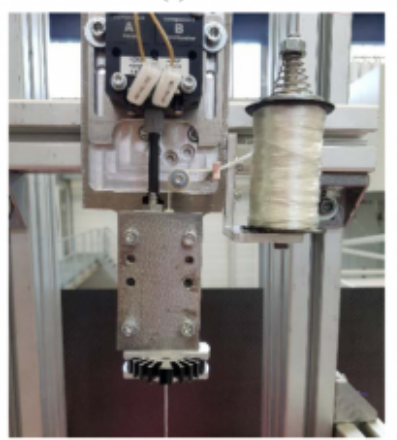

Fig. 2. Picture of test stand setup (a), schematic of the main components (b) and close up of the print head (c).

\section{Resin characterization - Viscosity and cure behavior of the neat resin}

The plate-plate measurements within the rheometer device resulted in an initial resin viscosity of $70 \mathrm{mPas}$, which is considered sufficiently low to realize fast impregnation of the selected glass fiber reinforcement while passing through the print head.

In order to define suitable photo-cure parameters, cure-temperature was varied from $25{ }^{\circ} \mathrm{C}$ to $100{ }^{\circ} \mathrm{C}$ and radiation time was varied from $1 \mathrm{~s}$ to $10 \mathrm{~s}$. Despite the difference in nominal wavelength between light source and photoinitiator, activation could be reliably achieved.

Fig. 3 shows the resulting storage modulus in dependence on time. Radiation and measurement were started simultaneously. At room temperature, a radiation time of $1 \mathrm{~s}$ can be assumed as too short for an immediate conversion to a polymer network. If the resin is exposed to the light source for $5 \mathrm{~s}$ or longer, the storage modulus increases significantly faster. A rise in cure-temperature results in an even further increased cure rate and a higher level of stiffness, which indicates an increased degree of cure. Based on the continuously advancing storage modulus, a darkcure effect can be observed at all measurements. 
(a)

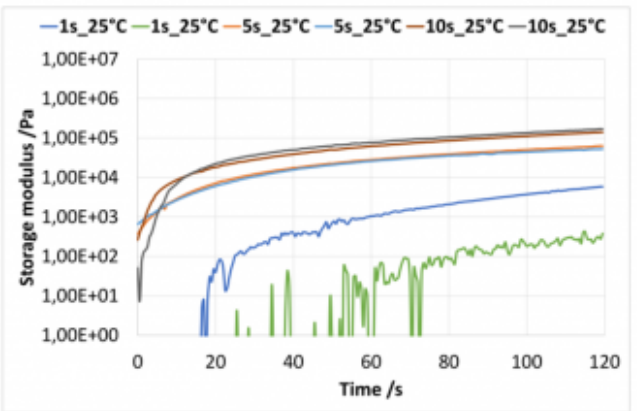

(b)

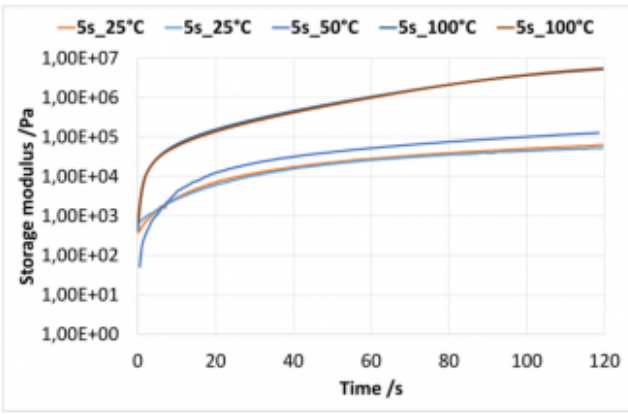

Fig. 3. Results of the UV-rheological measurements (a) at different radiation periods and (b) test temperatures.

In order to evaluate the adjusted cure state, the results of the DMA measurements using the SRF can be consulted. The pre-cured specimens ( $5 \mathrm{~s}$ radiation at room temperature) were stored at room temperature for several days to allow complete dark-cure. Fig. 4 shows the temperature-dependent storage and loss modulus of the pre- and post-cured neat resin specimen. The determination of the glass transition by evaluating the inflection point of G' reveals a first $\mathrm{T}_{\mathrm{g}}$ around $65{ }^{\circ} \mathrm{C}$ for the pre-cured resin. The same measurement reveals a second glass transition around $120{ }^{\circ} \mathrm{C}$. It can be assumed that this represents the final $\mathrm{T}_{\mathrm{g}}$ as it is reappears during the second heating. Furthermore, the post-cured specimen has a higher storage modulus, which confirms the previously mentioned assumption of cure-dependent stiffness.

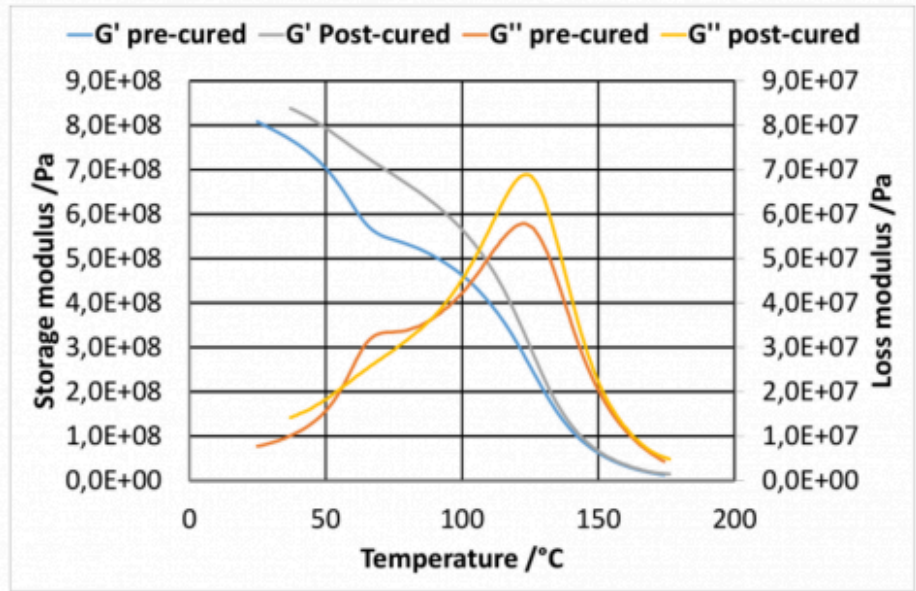

Fig. 4. Results of the DMA measurements on a pre-cured neat resin specimen and subsequent measurement using the thermally post-cured specimen.

\section{Parametric studies and composite characterization}

\subsection{Thermal imaging analysis}

Thermographic images were taken under stationary process conditions resulting in temperature profiles along the roving direction. In order to draw conclusions about the reaction behavior, the printing velocity and the light intensities 
Experimental studies for the additive manufacturing of continuous fiber reinforced comp...

(distance of light source to roving) were varied. Fig. 5 shows an exemplary thermal image of a fiber strand. Based on the pre-defined printing velocity the data can be converted from location- to time-dependent temperature curves (Fig. 6).

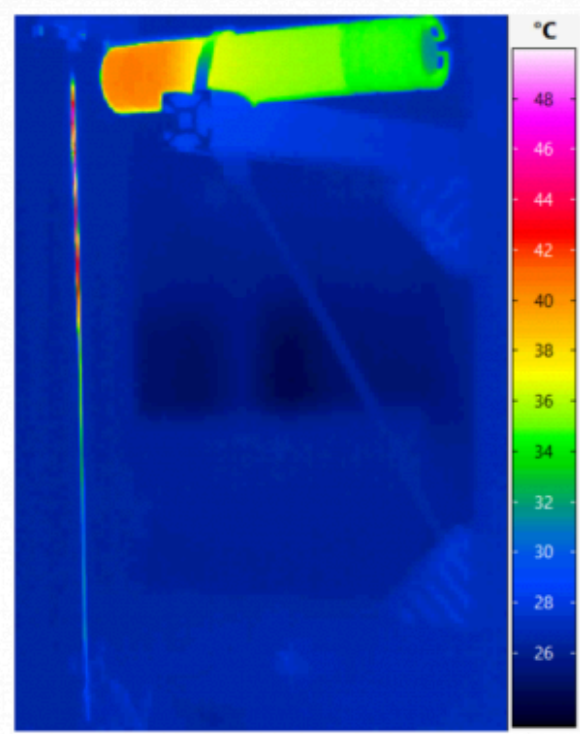

Fig. 5. Exemplary thermal image of the impregnated roving while exposed to the UV-light source.

Fig. 6 shows the resulting temperature profiles that will be discussed in analogy to the expected cure rate. Generation of exothermal heat is observed immediately after radiation has started, which is marked by the first dot on the curve. Temperature increase and reactivity slow down as soon as the fiber strand leaves the exposure zone, which is marked by a second dot. A printing velocity of $4.6 \mathrm{~mm} / \mathrm{s}$ and a UV-light distance of $7.5 \mathrm{~mm}$ lead to a premature temperature decrease, which could be reproduced by several measurements. In general, the maximum temperature $\mathrm{T}_{\max }$ is more pronounced at lower printing velocities. With increasing printing velocity, $\mathrm{T}$ max is reached earlier and the temperature equilibrates faster.

It can be concluded, that the initial reaction rate does not depend on the printing velocity, because the initial slope of the curves is identical for a fixed UV-light distance (Fig. 6 a, b and c). In contrast, a decreasing light intensity, leads to a decrease of the slope, as well as a reduced maximum temperature. Hence, it is expected that a shorter exposure time and less light intensity lead to a lower degree of cure. Matrix that was still tacky after very short exposure to UV-light was observed to solidify after some time spent at room temperature and ambient light due to the dark-cure effect of the resin.

(a)

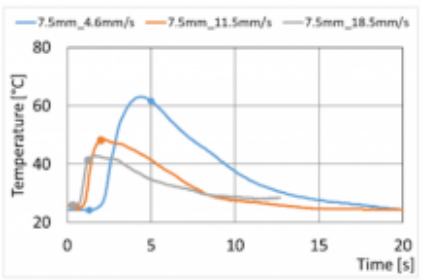

(b)

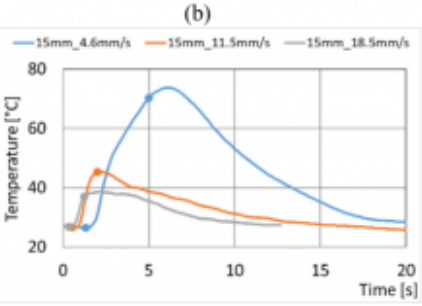

(c)

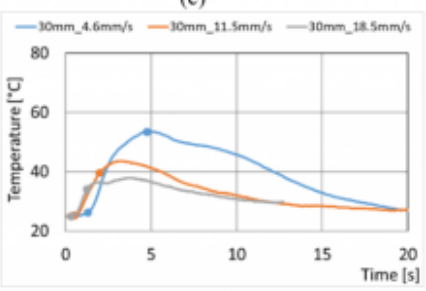

Fig. 6. Roving temperature over time for varying distance of the UV-light source: (a) $7.5 \mathrm{~mm}$ (b) $15 \mathrm{~mm}$ and (c) 30 
$\mathrm{mm}$; dots mark the beginning and the and of exposure to UV-light.

\subsection{Thermomechanical Tests}

The samples that were manufactured during the thermal imaging experiments described above were subjected to DMA using SCF after completion of dark-cure at room temperature. Again the glass transition temperature $\mathrm{T} g$ was determined by evaluating the inflection point of the storage modulus G', Fig. 7.

(a)

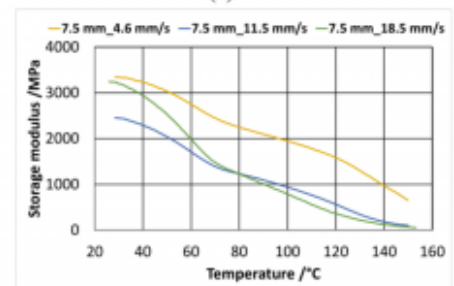

(b)

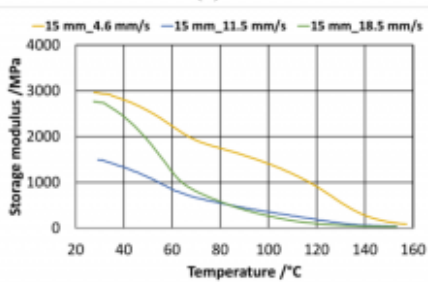

(c)

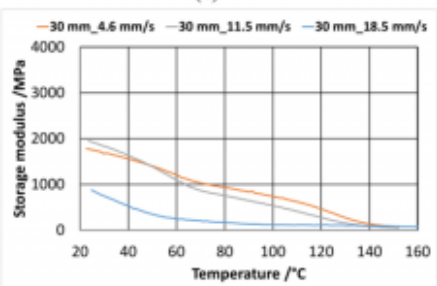

Fig. 7. Results of DMA measurements of impregnated fiber reinforced composite specimens originating from the developed printing process exposed to different light intensities for vary printing velocities - distance of light source to sample (a) $7.5 \mathrm{~mm}$, (b) $15 \mathrm{~mm}$ and (c) $30 \mathrm{~mm}$.

By comparing the maximum temperature $\mathrm{T}_{\max }$ that was reached during cure of the composite material and the $\mathrm{T}_{\mathrm{g}}$ determined by DMA (Fig. 8), it can be concluded that higher process temperatures lead to an higher degree of cure. These results are in accordance with the previously described neat resin characterization. Hence, a direct correlation between process parameters and the cure state can be derived.

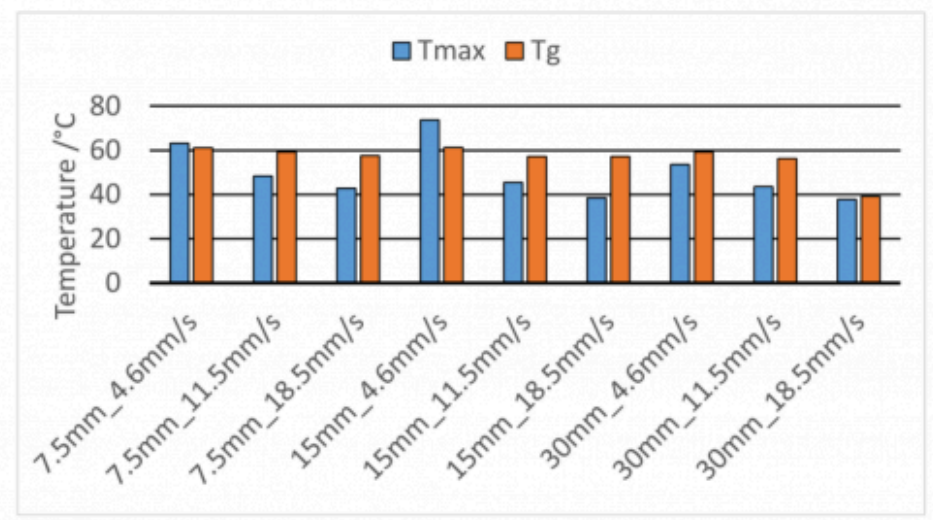

Fig. 8. Glass transition temperature of samples originating from the printing process vs. peak process temperature measured with thermal imaging.

\section{3D-Printing-process and manufacturing cell}

For the automated deposition of straight and curved path patterns and the analysis of process-structure-property relations of UV-cured and 3D-printed FRP, a manufacturing cell with a reasonable design space is required. In relation to a targeted design space of $330 \mathrm{~mm}$ x $330 \mathrm{~mm}$ x $300 \mathrm{~mm}$, the print head presented in section 2.3 is rather long (580 
Experimental studies for the additive manufacturing of continuous fiber reinforced comp...

$\mathrm{mm}$ ) and thin. A fixed print head setup with a build platform guided by a KUKA KR 6 industrial robot was chosen (Fig. 9). Advantages of this design are the reduced moving mass and the stationary routing of the supply lines. The manufacturing cell was realized out of modular aluminum construction profiles. External access to all process controls is ensured. The print head is fixed at a central column, while the robot is placed laterally in order to fully exploit its range of motion. The modular design allows future adjustments of the setup.

For printing parts with adjustable composite properties, an integration of the UV-light source into the print head is required. Furthermore, the distance between radiation zone and deposition nozzle needs to be as short as possible. These requirements were met by using exchangeable LED modules with different wavelength ranges that were mounted on the printing head. The adjustable power output of the LED modules allows further fine tuning of the radiation conditions.

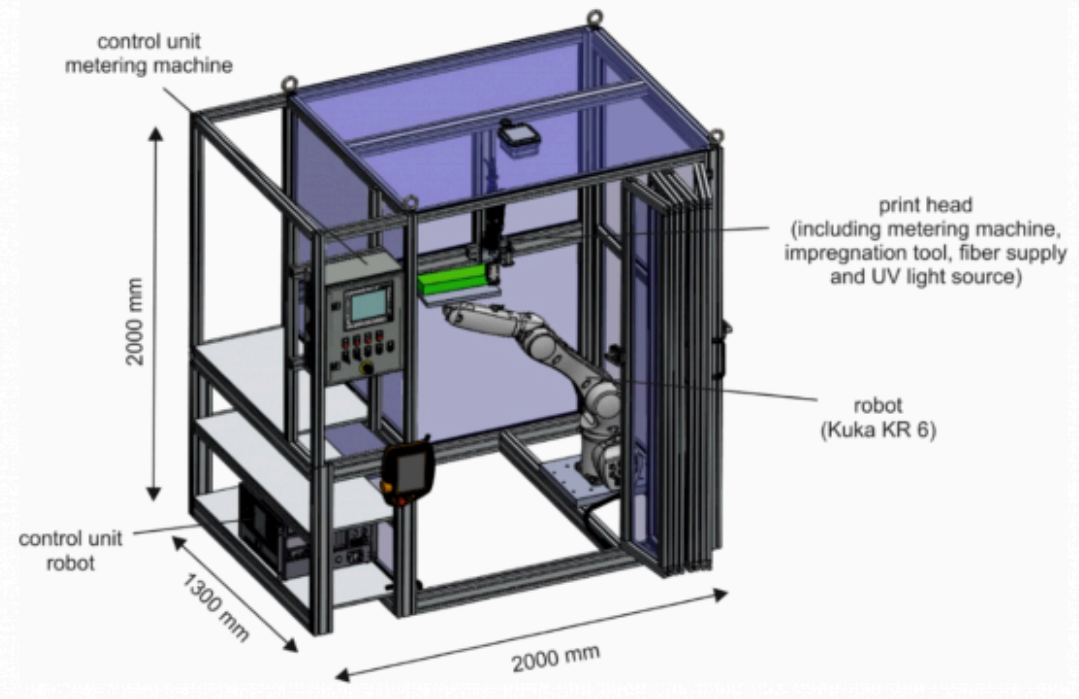

Fig. 9. Manufacturing cell for thermoset 3D-printing process with continuous fiber reinforcement.

\section{Conclusion}

The additive manufacturing of continuous fiber reinforced composites represents a promising approach for flexible production of future resource-efficient and load-adapted lightweight structures. Thermoset UV curable matrix materials are highly interesting for this purpose as they provide high-performance thermo-mechanical properties. In order to realize such a process route, complete fiber impregnation, fast cure and reliable process monitoring must be ensured.

The presented work comprises (1) resin selection and characterization, (2) print head design and implementation as well as (3) parametric studies of the process. Special focus is put on the sub-process steps of impregnation and cure. It was found that the initial viscosity of the selected epoxy resin is sufficiently low to impregnate a glass fiber roving within few seconds. Photo-initiation of the curing reaction was analyzed by oscillating UV-rheological measurements yielding a clearly defined process window. A radiation period between $1 \mathrm{~s}$ and $5 \mathrm{~s}$ at room temperature was found suitable to activate cure. The cure rate can be further accelerated by an increased temperature. DMA experiments with pre-cured neat resin material demonstrated the possibility of thermal post- cure. The gathered information was subsequently transferred to parametric studies using a self-developed printing head. A glass fiber roving was successfully impregnated and photo-cured at printing velocities from $4.6 \mathrm{~mm} / \mathrm{s}$ to $18.5 \mathrm{~mm} / \mathrm{s}$. Thermal imaging was 
used to monitor the exothermal heat generated by the crosslinking reaction. The manufactured specimens were used for DMA measurements that confirmed the previously determined pre- and post-cure behavior of the resin.

\section{Acknowledgements}

We thank Sächsische Aufbaubank - Förderbank - (SAB) for funding this research within the framework of Fraunhofer Leistungszentrum "Smart Production and Materials". This research is co-financed with tax funds on the basis of the budget approved by the Saxon State Parliament.

[1] Kellens K, Baumers M, Gutowski TG, Flanagan W, Lifset R, Duflou JR. Environmental Dimensions of Additive Manufacturing: Mapping Application Domains and Their Environmental Implications. Journal of Industrial Ecology 2017;21(S1):S49-S68. https://doi.org/10.1111/jiec.12629.

[2] Wang X, Jiang M, Zhou Z, Gou J, Hui D. 3D printing of polymer matrix composites: A review and prospective. Composites Part B: Engineering 2017;110:442-58. https://doi.org/10.1016/j.compositesb.2016.11.034.

[3] Goh GD, Yap YL, Agarwala S, Yeong WY. Recent Progress in Additive Manufacturing of Fiber Reinforced Polymer Composite. Adv. Mater. Technol. 2019;4(1):1800271. https://doi.org/10.1002/admt.201800271.

[4] Parandoush P, Lin D. A review on additive manufacturing of polymer-fiber composites. Composite Structures 2017;182:36-53. https://doi.org/10.1016/j.compstruct.2017.08.088.

[5] Tian X, Liu T, Yang C, Wang Q, Li D. Interface and performance of 3D printed continuous carbon fiber reinforced PLA composites. Composites Part A: Applied Science and Manufacturing 2016;88:198-205. https://doi.org/10.1016/ j.compositesa.2016.05.032.

[6] van Der Klift F, Koga Y, Todoroki A, Ueda M, Hirano Y, Matsuzaki R. 3D Printing of Continuous Carbon Fibre Reinforced Thermo-Plastic (CFRTP) Tensile Test Specimens. OJCM 2016;06(01):18-27. https://doi.org/10.4236/ ojcm.2016.61003.

[7] Matsuzaki R, Ueda M, Namiki M, Jeong T-K, Asahara H, Horiguchi K et al. Three-dimensional printing of continuousfiber composites by in-nozzle impregnation. Sci Rep 2016;6:23058. https://doi.org/10.1038/srep23058.

[8] Tyler KL, Stockett RC. Additive Manufacturing System Having In-Situ Reinforcement Fabrication(WO 2018/ 140418A1).

[9] Natale G, Levi M, Postiglione G. Apparatus and method for three-dimensional printing of continuous fibre composite materials(W02017085649A1); 2017.

[10] Adumitroaie A, Antonov F, Khaziev A, Azarov A, Golubev M, Vasiliev VV. Novel Continuous Fiber Bi-Matrix Composite 3-D Printing Technology. Materials (Basel) 2019;12(18)

PDF automatically generated on 2021-05-25 10:19:48

Article url: https://popups.uliege.be/esaform21/index.php?id=3807

published by ULiège Library in Open Access under the terms and conditions of the CC-BY License (https://creativecommons.org/licenses/by/4.0) 